\title{
Technical Highlights of the Recent Literature
}

1002 Uses for Biotin....and Counting

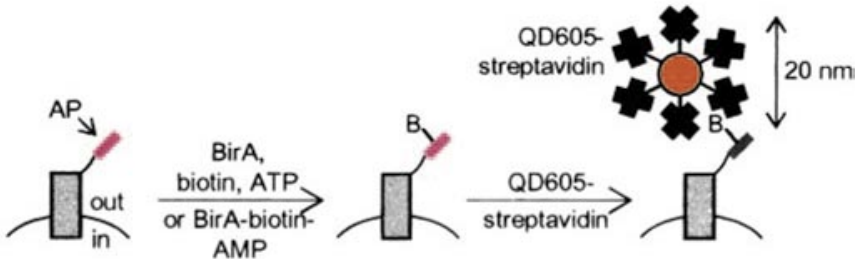

Reprinted with permission. @ 2005 National Academy of Sciences, USA

Just when you thought there couldn't possibly be any more uses for the ubiquitous and pliable streptavidinbiotin system...think again. In an effort to improve upon current methods for studying membrane protein trafficking, Howarth et al. have come up with a creative collaboration of the small 15-amino acid biotin acceptor peptide (AP) and streptavidin-conjugated quantum dots (QDs). Proteins tagged with AP, expressed and tested in various cell types, could be rapidly biotinylated by addition of recombinant Escherichia coli biotin ligase, biotin, and ATP; addition of streptavidin-QD conjugates completed the surface labeling procedure. The authors show that primary neuronal cells, often sensitive to transfection and difficult to manipulate, could be straightforwardly labeled, and the targeted receptor tracked, even down to single molecules. Although QDs are somewhat bulky and are shown to prevent access to certain parts of the cell membrane, they are still an improvement over complexes of multiple antibodies currently used in receptor trafficking studies. Moreover, the rapidity with which AP-tagged proteins could be labeled (in the order of minutes) should allow for dramatically improved time resolution in pulse-chase studies, while the stability of the streptavidin-biotin interaction-and its specificity-means that long-term imaging studies should be possible. In addition to the clear advantages presented in this paper, the technique is certainly more widely applicable. AP, being a smaller tag, should be less disruptive to protein folding and protein-protein interactions than bigger tags such as myc and GFP. Its future use in pulse-chase experiments should reduce the occurrence of aberrant results caused by the presence of large antibody complexes. - SS

Howarth et al. 2005. Targeting quantum dots to surface proteins in living cells with biotin ligase. Proceedings of the National Academy of Sciences of the USA 102:7583-7588

\section{Master Manipulator}

Ever enviously watched someone with a magnetic personality bend the behavior and actions of others? Although foolproof techniques for exerting a Svengali-like influence in social interactions remain to be found, if you're willing to confine your aspirations to the microscopic world, look no further than a recent report by Tanase et al. The study describes a novel approach to the problem of convincing cells to arrange themselves in a desired micropattern. Cells patterned in this way can be used for miniaturized biosensors or for a detailed examination of mechanical forces involved in cell adhesion and activity. One established way of organizing cells involves using a substrate bearing cell-adhesive ligands in predefined areas, but this is a slow (and generally irreversible) process. Alternatively, cells can be coaxed into position with laser energy or electric fields. In this paper, Tanase et al. describe how magnetic fields can be used for the same purpose. In their strategy, cells become susceptible to magnetic manipulation because they are bound with ferromagnetic nickel nanowires. In the simplest scenario, nanowire-bearing 3T3s are exposed to a uniform, low-frequency magnetic field, causing them to self-assemble into chains. More sophisticated patterns are possible by using a flow chamber in which the base consists of a

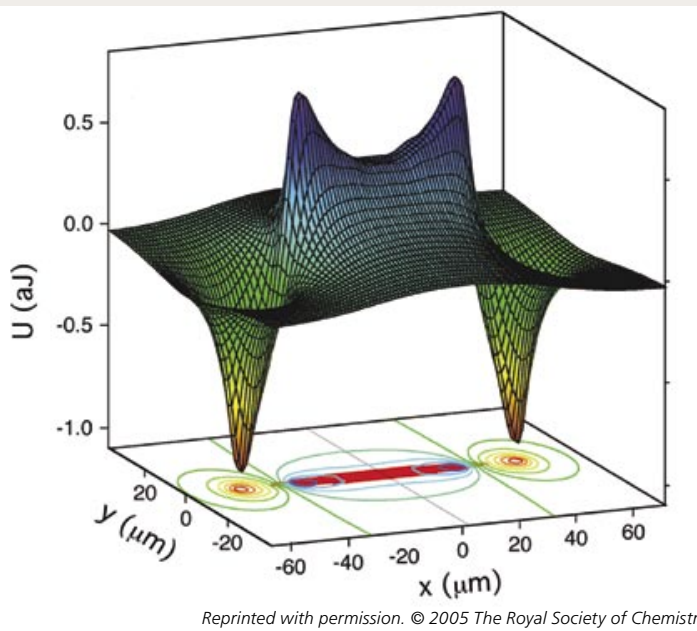

Reprinted with permission. @ 2005 The Royal Society of Chemistry. glass slide that has up to 4000 elliptical micromagnets arrayed upon it. The magnetic forces (which can be modeled accurately) draw the nanowire-associated fibroblasts to the poles of the oblong micromagnet and away from its center. Varying the array element spacing and the fluid flow parameters allows different cell patterns to be formed. Under certain conditions, sparse arrays suitable for applications requiring spatially separated cells form; with other settings, cell chains predominate instead. Given its elegance, this flexible, tunable method of magnetic cell positioning should soon be attracting a lot of cells-and researchers. —ND Tanase et al. Assembly of multicellular constructs and microarrays of cells using magnetic nanowires. Lab on a Chip 5(6):598-605 (2005) 


\section{ChIPping Away at the Genome}

It is a truism that in most fields of scientific endeavor, when a particular question is answered, a multiplicity of new questions arise. This paradigm, reminiscent of many MC Escher sketches, is particularly true of the recent completion

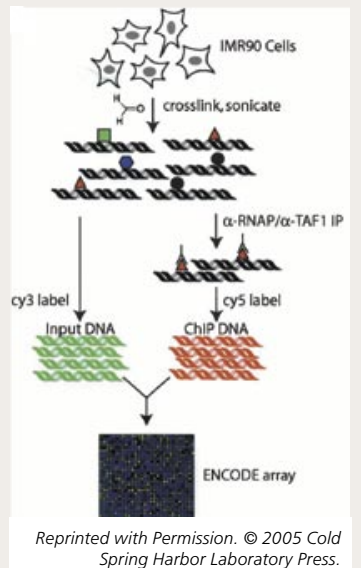

Spring Harbor Laboratory Press.

are confident that this met of the human genome sequencing project. A variety of computer-based techniques, often requiring complex algorithms and considerable processing power, have been developed to scan the raw sequence data in an attempt to identify true coding sequences, estimated to make up only less than $2 \%$ of the genome. Taking a more "natural" approach in a recent Genome Research article, Kim et al. harness the cell's own promoter recognition proteins to point the way to putative open reading frames. Adapting chromosome immunoprecipitation (ChIP) technology and targeting the pre-initiation complex (PIC) that binds active promoters, the authors used antibodies against both RNA polymerase (RNAP) and a TFIID transcription factor subunit (TAF1) to pull down cross-linked DNA fragments. Being somewhat conservative, analysis was limited to those sequences bound by both RNAP and TAF1, a total of 118 putative promoters. Utilizing RefSeq and GenBank ${ }^{\circledR}$ database scans, in vitro reporter assays, and a modified RACE technique, called ARLM-RACE, a full $90 \%$ of PIC-binding sequences could be assigned to known CDNA transcripts or shown to drive transcription of as yet undefined RNAs. The work was extended to three additional cell lines and, although the experiments were not as extensive, the results were highly comparable to the initial findings. The authors binding sites. - SS

Kim et al. 2005. Direct isolation and identification of promoters in the human genome. Genome Research [Epub ahead of print May 17, 2005]

\section{¡Azúcar!}

That passionate cry of "sugar" was a trademark of the late Celia Cruz, evoking the despair of sugar-plantation slaves but also defiantly proclaiming the singer's passionate dedication to her craft. It's a bittersweet sentiment glycomics researchers might understand, given that biochemical analysis of protein-linked oligosaccharides is a painstaking and energy-intensive process. Glycosylation is not hard-wired in the sense of nucleic acid or protein synthesis; although potential glycosylation sites can be predicted, this gives no hints about the structure of any glycan linked at this position. At the same time, glycosylation profoundly affects the function of therapeutically relevant glycoproteins such as recombinant monoclonal antibodies. Therefore, using glycosylated biologics in drug discovery and manufacturing them in controlled conditions require sophisticated and accurate methods for measuring and characterizing glycans-all without using

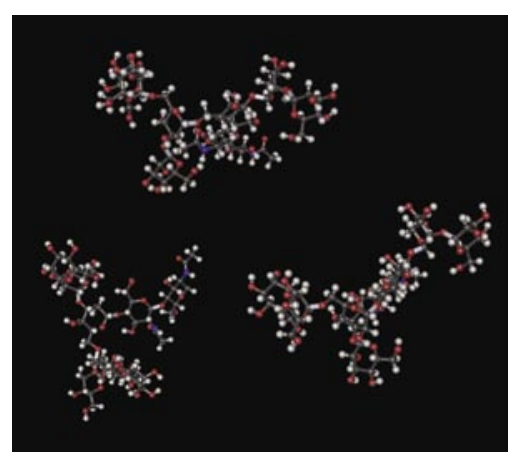
significant amounts of the analyte. To meet these demanding needs, Novotny and colleagues describe a state-of-the-art workflow for detailed structural investigation of recombinant glycoproteins, in this case, a murine monoclonal antibody. For quantitative analysis of the monosaccharides present, the authors adapted an existing technique involving monosaccharide release and derivativization with the labeling agent APTS to render the subunits detectable by capillary electrophoresis/laser-induced fluorescence (CE-LIF). A complementary CELIF approach analyzes the intact glycans (which are enzymatically released); comparison of the resulting electropherograms with patterns from oligosaccharide standards provides a profile of the structural forms present. More detailed structural information is possible with MALDI-TOF MS analysis of the liberated glycans and enzymatic sequencingthat is, sequential treatment with the various exoglycosidases. Mapping of the glycosylation sites was performed by trypsin digestion, microcolumn liquid chromatography, electrospray ionization, and mass spectrometry using a QTOF instrument. MS/MS was performed when diagnostic carbohydrate ions were detected, thus providing information about the glycosylation sites and the glycan structure itself. Combining multiple methodologies in this way yields qualitative and quantitative information about glycans and requires less than $20 \mu \mathrm{g}$ of glycoprotein, creating an efficient and sensitive means of validating glycosylated biologics. This new workflow should sweeten the work of those involved in oligosaccharide characterization of biopharmaceuticals-and that too is worth shouting about. —ND Mechref et al. Comprehensive assessment of $N$-glycans derived from a murine monoclonal antibody: a case for multimethodological approach. Electrophoresis 26(10):2034-2046 (2005) 